\title{
Research samples from families with genetic diseases: a proposed code of conduct
}

\author{
Peter S Harper
}

Research on samples from families with genetic disease underlies many of the major advances that are occurring in medical genetics. But ethical and practical problems may arise when samples from relatives who are healthy but at risk are included in such studies. In particular, new molecular tests for specific gene mutations may result in the detection of a genetic defect in relatives who had neither expected this possibility nor given specific consent to such testing. Family members at risk should not be included in such studies unless strictly necessary, and in such cases specific consent should be obtained and information should be given about the implications of an abnormal result of a test. This is particularly important when stored samples from previous studies without such implications are being reused and is also relevant to the genetic testing of samples taken primarily for epidemiological studies of disorders when only a small proportion of cases is thought to be genetic in origin. There is a need for guidelines to protect both subjects and investigators in a field which is spreading rapidly and involving many clinical and laboratory research workers previously unfamiliar with genetic testing.

Over the past decade developments in molecular genetics have allowed the mapping and isolation of a large number of genes for serious human disorders; DNA analysis of specific mutations or of linked markers now gives the possibility of presymptomatic and prenatal diagnosis, of detection of healthy heterozygous carriers, and, in some instances, of inferring the severity of the disease from the nature of the mutation detected. Much of the research leading to these and other important advances has entailed the study of families with specific inherited disorders; samples from these families have provided an essential resource and will continue to do so.

Most samples taken for DNA analysis are of venous blood because of ease of sampling, minimal discomfort to the subject, and the large number of analyses possible on a single sample. Transformed cell lines are often established on particularly important samples, but more often the isolated DNA is stored at -20 or $-70^{\circ} \mathrm{C}$, providing a resource that can be used repeatedly without having to cause disturbance or discomfort by taking further samples.

Many research workers, both clinicians and laboratory scientists, maintain large collections of blood samples from patients with genetic disorders and their relatives. So far they have given little thought to the dangers of this practice as opposed to the advantages. Until recently I was as unaware of the potential pitfalls as most others but recent experience in providing a service of molecular diagnosis and prediction for several disorders, in particular a programme of presymptomatic testing for Huntington's disease, has shown that the dangers of misuse of research samples are real and likely to increase as more disease genes are isolated.

Indeed I am conscious that my awareness of most of the errors and problems described here is a direct result of having encountered them myself by not paying adequate attention to the points now recommended. This paper outlines some of the main problems and makes some suggestions for avoiding them. After discussion and modification they might form the basis of a code of conduct that could help both investigators and families with genetic diseases.

As most of the points raised here are self evident it is perhaps surprising that they do not seem to have been specifically raised in print, especially as other ethical issues in genetics have received extensive debate and publicity. Thus the ethics of gene therapy has been the subject of a special report,' as has the research use of fetal material ${ }^{2}$ and human fertilisation and embryology. ${ }^{3}$ By contrast the use of research samples for genetic tests receives no mention in the recent Medical Research Council booklet on responsibility in investigations on human participants ${ }^{4}$ or in the Royal College of Physicians' report on ethical issues in medical genetics'; the United States bibliography on ethical, legal, and social implications of the human genome project $^{6}$ contains no entry (among a total of around 2400) that seems related to the subject.

\section{What type of samples cause problems?}

It is important to distinguish between the different categories of samples stored as the potential for problems and for misuse varies widely between them. Service DNA banks, where samples are deposited specifically for service use, are likely to be a problem only when research and service activities are carried out in the same laboratory and when a clear distinction is not made between the two categories. ${ }^{7}$ Anonymous or strictly coded samples forming part of epidemiological studies are likewise not an issue, unless the worker proposes to break the code that protects an individual patient's identity. The principal concern is with identifiable samples taken from members of individual families for research purposes.

Within this group one can again distinguish between those affected with the disorder and those apparently unaffected but at risk. It is in this last group that most of the problems lie. Samples from affected patients are unlikely to cause difficulties unless they are taken from patients whose disorder has not previously been recognised as having a genetic basis (see below).

\section{What problems can arise?}

Most molecular studies of families with genetic diseases initially entail a genetic linkage approach by relying on a comparison of marker genotypes between 
affected and unaffected subjects to establish linkage with a particular marker. Once the gene is isolated a comparative approach is again important to establish that any change identified in the gene is in fact specific to affected subjects and not a harmless polymorphism or other coincidental change.

Inevitably such an approach will from time to time detect the genetic defect (or high risk marker) in a subject considered to be unaffected but who in fact may be very mildly affected in a variable disorder (for example, myotonic dystrophy) or who may develop the condition later in a disorder that is strongly age dependent (for example, Huntington's disease). In yet other disorders a proportion of known carriers of the gene never develop the disease (for example,

\section{"The problem of unexpected detection of genetic defects is only important in disorders of relatively late onset or variable phenotype."}

dominantly inherited retinitis pigmentosa, familial amyloidosis), so the subject detected may fall into this category.

The problem of unexpected detection of genetic defects is only important in disorders of relatively late onset or variable phenotype such as those mentioned above; commonly these are autosomal dominant in inheritance. For disorders of consistently early onset or static nature (for example, achondroplasia) such a case would not be likely to arise.

\section{What are the adverse effects of unexpected detection?}

The diagnosis of any serious genetic disorder is commonly accompanied by psychological upset, relating not only to the potential clinical effects of the disease but also to the genetic consequences. ${ }^{8}$ The importance of careful preparation, counselling, and support has been shown in genetic prediction for Huntington's disease, when adverse effects have in general been minimised by such measures. ${ }^{910}$ The unexpected detection of such a defect in a subject who has not specifically asked for this does not permit such a process.

A number of more specific problems may be created for the person concerned. These include implications for life and health insurance ${ }^{11}$ or for employment (if the finding is disclosed), and the genetic consequences for children, born or unborn, may have serious repercussions on marriage and family relationships. Uncertainty about the outcome may be a very real problem when the consequences of a disorder or a specific mutation are variable or incompletely known.

\section{Problems of consent}

Many of the problems mentioned above are aggravated or even created by uncertainties relating to consent. When the taking of a blood sample has been the only procedure, written consent may not have been obtained and it may be uncertain what consent was given for. Even written consent may be so limited or non-specific as to be of little use. When samples are stored successive phases of research may use the same sample, starting with attempts to localise a gene and continuing through its isolation to the study of mutations. Implications for the use of samples may exist at this later stage that were not present or even thought of when the sample was originally taken. This raises the question as to how different the work has to be before a separate and specific consent is sought from the person who provided the sample.

As approval from an ethical review committee has to be obtained before a research project can proceed it might be thought that this process would ensure that suitable consent was indeed obtained. Members of ethical committees, however, are often not experts in genetics and are likely to be more concerned with the dangers and discomfort of procedures or treatments than with the consequences of genetic information. Thus, a project entailing a simple venepuncture may pass review easily in comparison with an invasive measure without full consideration being given to the serious potential effects of detection of a genetic defect.

A particular difficulty that can arise when an abnormality is found is whether or not the person is expecting to receive a result from the sample taken. As the outcome of any research project is necessarily uncertain it is preferable to make it clear to any healthy member of a family from the outset that no result will be forthcoming either for themselves or for their doctor or medical record. This should resolve any later uncertainty. The situation may be different for samples from subjects affected by a known genetic disorder, when specific information such as type or nature of a mutation may be helpful and valuable.

\section{Problems for the investigator}

A problem is most often first recognised by the research worker when a sample from a person at risk but considered healthy is analysed and shows the specific gene defect expected only in affected subjects. What, if anything, should be done? Should the information be given to the person who provided the sample and, if so, how? Should their doctor be informed or the information be recorded in the person's medical record? Or should the research laboratory and clinical research worker keep the information to themselves or even destroy it?

Depending on the nature of the disease and the attitude of the investigator this question may receive varying answers. Many research workers feel strongly that they should do everything possible to help those who take part in research and that it is wrong to withhold information that could be important. This may especially be the case when the clinical research worker is also concerned in providing services.

The lack of clarity regarding consent, however, will often mean that the expectations and likely reactions of the subject will be uncertain or unknown and the process by which the information came to light will itself make any associated preparation and counselling difficult. The alternative of not disclosing the information but keeping the result in the laboratory may seem easier initially but also poses difficulties.

\section{"The importance of careful preparation, counselling, and support has been shown in genetic prediction for Huntington's disease."}

It may prove very hard to keep the information completely contained; later workers may not realise it had not been disclosed while, if the research worker also has clinical contact with the family, the possession of such knowledge may produce an unacceptable dilemma, something not altered even if the information or identifying details are destroyed. The legal situation regarding non-disclosure of relevant results of research is also questionable.

The reason why it is usually impossible to resolve 
this dilemma satisfactorily is that the true origin of the problem lies further back - that is, the sample should not have been tested in the first place, at least not without specific permission and a clear understanding of the implications of the testing. Once the test has been done and an abnormal result obtained the problem has been created; any subsequent measures are more in the nature of damage limitation than a true solution.

In fact the use of samples from healthy but at risk members of a family is often unnecessary for molecular genetic research. Such samples may commonly confuse genetic linkage analysis. That they are taken at all often implies lack of thought; relatives may often volunteer to provide a sample feeling that it must be of help or a family may simply be sampled en masse at a home visit. The serious consequences of such sampling may be apparent only much later. In a non-progressive disorder the discovery of a mutation thought to be pathogenetic in a proportion of healthy relatives may be of value in showing that the gene is not fully penetrant, but the genetic implications still necessitate information being given on this possibility before such samples are tested.

Genetic research if the affected subject is not known to have an inherited disorder

The discovery of relevant mutations in samples from subjects not known to have inherited disorders is likely to increase sharply as specific genes in common nonmendelian conditions become identified. Already genetic subgroups are being identified in disorders previously not considered as genetic; important examples include mutations in the gene for $\beta$ amyloid protein in Alzheimer's disease, ${ }^{12}$ prion mutations in Jakob-Creutzfeldt dementia, ${ }^{13}$ and p53 mutations in multiple cancer syndromes. ${ }^{14}$ Although initially detected in rare families showing mendelian inheritance, these mutations are now being searched for and

\section{"A project entailing a simple venepuncture may pass [ethical] review... without full consideration being given to the serious potential effects of detection of a genetic defect."}

detected in series of cases without an obvious family history.

The discovery of such a mutation poses grave consequences for relatives as well as for individual subjects by raising the question of whether the possibility of a genetic basis of the disorder being discovered, along with its consequences, was discussed and consented to at the time the sample was taken. It may occasionally be argued that a finding of this nature should not be withheld from relatives who may be at genetic risk as a result. Such a conclusion would seem to make it even more necessary for information to be given in advance that such an outcome might be possible. If the study has re-used samples taken originally for non-genetic research additional consent would seem to be essential.

\section{A tentative code of conduct}

The problems involving research samples from families with genetic disorders are real and increasing; if not recognised and avoided they are likely to harm both the individual subjects detected and possibly the investigator. Can guidelines be suggested that will prevent problems arising and, at the same time, avoid unnecessary bureaucracy for those undertaking research? The following suggestions are made as a basis for further discussion.

(1) Family members "at risk" should not be sampled unless this is strictly necessary for the research, especially in late onset or variable disorders. This applies particularly to children. When unaffected subjects are included in a study the proposal should justify this and should include a clear plan as to what will be done in the event of a genotypic abnormality being detected.

\section{"The discovery of a mutation raises the question of whether the possibility of a genetic basis... was discussed and consented to at the time the sample was taken."}

(2) When consent is given for sampling by such a person it should specifically be made clear that the person's risk will not normally be modified and that no result in relation to this should be expected; also that no such result will be sent to their doctor or placed in their medical record unless this has been specifically requested.

(3) If the sample is to be stored and used for future tests new consent should be obtained if the implications for the person at risk resulting from the new research are considerably different-for example, if mutations are to be looked for rather than a general linkage analysis.

(4) If the possibility of identifying defects in people at risk is foreseen and inevitable then such samples should be coded or made anonymous for the purpose of these tests unless the person concerned.has specifically requested that relevant information should be disclosed and has received information that allows the implications to be fully understood.

(5) If a person at risk giving a research sample later requests presymptomatic testing or other genetic services a new sample should be taken and the request handled in the same way as it would be for any other person making this request as a service.

(6) When a test that may show a specific genetic defect is proposed on people affected by a disorder not previously known to be genetic the possible genetic implications should be made clear and new consent obtained if previously taken samples are being restudied.

(7) Ethical committees should pay at least as much attention to the consequences of a sample being taken as to the risks attached to the sampling procedure.

\section{Conclusion}

The power of the developments in molecular genetics is such that it is no longer adequate to rely on implied or general consent if genetic analysis is to be carried out on samples from family members at risk of a genetic disorder. The implications of finding an unexpected abnormality in a healthy relative are too potentially serious to make it wise to use samples except in a carefully controlled way: if such an abnormal result is likely to be obtained investigators should attempt to ensure anonymity of the sample so as to protect both the person at risk and themselves.

The steps suggested here will inevitably entail some extra effort in terms of consent and explanation at the time of sampling, but the adverse consequences of not following this course are likely in the long term greatly to outweigh the work involved. The adoption of a code 
of conduct in relation to genetic research of this type should not only help to avoid specific problems of the type described but also help to maintain trust between families and research workers. That trust has been the basis of much of the successful research so far and must be maintained in the future if genetic advances are to be continued and used to help families with serious inherited disorders.

1 Committee on the Ethics of Gene Therapy. Report. London: HMSO, 1992.

2 Review of the guidance on the research use of fetuses and fetal material. Repon London: HMSO, 1989.

3 Committee of Inquiry into Human Fertilisation and Embryology. Report. London: HMSO, 1984.

4 Medical Research Council. Responsibility in investigations on human participants and material and on personal information. London: Medical Research Council, 1992.

5 Royal College of Physicians. Erhical issues in clinical genetics. London: Royal College of Physicians, 1991.
6 United States Department of Energy. Bibliography: ethical, legal and social implications of the human genome project. Washington DC: US Department of Energy, 1992.

7 Yates JRW, Malcolm S, Read AP. Guidelines for DNA banking. $f$ Med Genet 1989;26:245-50.

8 Firth MA. Diagnosis of Duchenne muscular dystrophy: experience of parents of sufferers. $B M \mathcal{F}^{1}$ 1983;286:700-1.

9 Brandt J, Quaid K, Folstein SE, Garber P, Maestri NE, Abbot MH, et al. Presymptomatic diagnosis of delayed-onset disease with linked DNA markers: the experience in Huntington's disease. IMM 1989;261:3108-15.

10 Morris MJ, Tyler A, Lazarou L, Meredith L, Harper PS. Problems in genetic prediction for Huntington's disease. Lancet 1989;ii:601-3.

11 Harper PS. Genetic testing and insurance. $f R$ Coll Physicians Lond 1992;26:184-7.

12 Yankner BA, Mesulan MM. B-amyloid and the pathogenesis of Alzheimer's disease. N Engl f Med 1991;325:1849-57.

13 Prusiner SB. Molecular biology of prion disease. Science 1991;252:1515-22.

14 Srivastava S, Zou Z, Pirollo K, Blattner W, Chang EH. Germ-line transmission of a mutated p 53 gene in a cancer-prone family with Li-Fraumeni syndrome. Nature 1990;348:747-9.

(Accepted 9 March 1993)

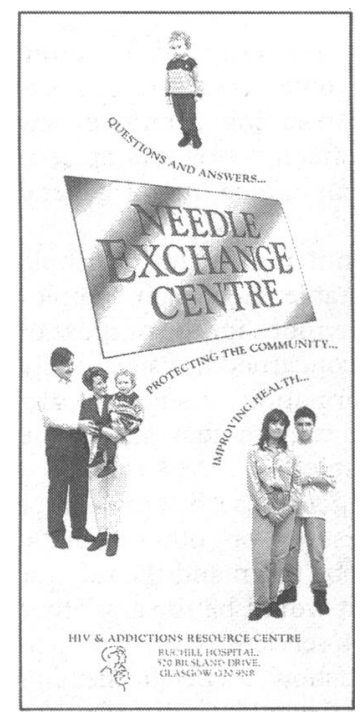

FIG 1-Leaflet produced to answer questions about needle exchanges

\title{
Building a city wide service for exchanging needles and syringes
}

\author{
Laurence Gruer, John Cameron, Lawrence Elliott
}

How best can injecting drug misusers obtain clean injecting equipment in a city where drug injecting is widespread? An exchange service for needles and syringes throughout Glasgow has been established in health centres and clinics in the evening. Over the past four years seven new exchanges have been opened and over 2700 injecting drug misusers have attended. Attendances rose from under 1000 in $\mathbf{1 9 8 8}$ to 28000 in 1992 . The exchanges also provide a wide range of other health and social services. Public hostility to the exchanges has abated. During the same period equipment sharing in the city diminished and the observed prevalence of HIV among injecting drug misusers stabilised at around $1 \%$.

Since 1987 an important part of the United Kingdom's strategy for preventing HIV infection has been the provision of sterile needles and syringes to discourage equipment sharing by drug misusers. ${ }^{1}$ The main sources of injecting equipment are community pharmacies and specialist needle exchanges. The former are cheap and informal with long opening hours but depend on the voluntary participation of the pharmacist and offer limited opportunities to provide clients with health and social services. Needle and syringe exchanges may stand alone or be part of a wider service to drug misusers. To succeed they need to be attractive and accessible. They should be close to where potential clients live and open at times they find convenient. ${ }^{2}$ In the United Kingdom, however, most needle exchange services have been operated from a single site during normal working hours. ${ }^{3}$

Glasgow is a sprawling city with a population of about 850000 . Drug injecting is common in many areas and is particularly prevalent in both deprived inner city districts and peripheral housing estates. In 1989 around 9400 people were injecting drugs, representing $1.5 \%$ of the population aged between 15 and $55 .{ }^{4} \mathrm{~A}$ pilot needle exchange began in June 1987 at Ruchill Hospital in the north of the city. The exchange opened twice weekly from 130 to $400 \mathrm{pm}$, and in line with Scottish Office guidelines three needles and syringes were offered at each visit. By December 1988 it was being attended by only $20-30$ clients each week, few of whom did so regularly. Clearly the exchange was not what most drug misusers wanted. Nor was it popular with the local community, many of whom thought it worsened the drug problem and increased the number of discarded needles. The only other legitimate source of injecting equipment was a few community pharmacists.

Given a reported $50 \%$ prevalence of HIV infection among injecting drug misusers in nearby Edinburgh ${ }^{5}$ and the diagnosis of many new cases in Glasgow, ${ }^{6}$ urgent action was required. We therefore sought to develop needle exchanges in local communities that would be acceptable to both drug misusers and the general public. Here we describe what happened over the next four years.

\section{Methods}

THE SERVICE MODEL

In January 1989 a second needle exchange was established at a drug project in a peripheral housing estate. It opened twice a week in the evenings and its success was immediate. Within a few weeks 50 clients were attending each evening. As the premises were clearly too small we asked whether the nearby health centre could be used in the evening when it was otherwise closed. Excellent facilities would thus be available without disrupting other services at the health centre. Agreement was reached and the health centre exchange opened twice weekly in May 1989 for a trial period of three months. During that time its operation was free of trouble and attendances reached 100 each evening. The opportunity to establish a network of similar exchanges within health centres and clinics throughout the city now clearly existed.

\section{ESTABLISHING NEW EXCHANGES}

It was judged that nine exchanges would ensure accessibility to drug misusers in the most seriously affected parts of the city. Once a site was selected extensive consultation was undertaken with management and staff in the premises, community organisations, local police, and social services. In most areas public meetings were held to explain the proposals and deal with anxieties and hostility, which were often deeply felt. In two areas a monitoring group of local residents was set up to examine the impact of the exchange on the community. Once local consent for an exchange had been achieved the necessary approval was sought from the minister of health at the Scottish Office.

From February 1991 health promotion officers were assigned to each operating or proposed exchange to develop community liaison. In April 1991 a leaflet designed to answer questions commonly raised about 